\title{
CCD photometric search for peculiar stars in open clusters
}

\section{Collinder 272, Pismis 20, Lyngå 14, NGC 6396 and NGC 6611^}

\author{
E. Paunzen ${ }^{1,2}$, O. I. Pintado ${ }^{3, \star \star}$, and H. M. Maitzen ${ }^{1}$ \\ 1 Institut für Astronomie der Universität Wien, Türkenschanzstr. 17, 1180 Wien, Austria \\ 2 Zentraler Informatikdienst der Universität Wien, Universitätsstr. 7, 1010 Wien, Austria \\ 3 Departamento de Física, Facultad de Ciencias Exactas y Tecnología, Universidad Nacional de Tucumán, Argentina - Consejo \\ Nacional de Investigaciones Científicas y Técnicas de la República Argentina
}

Received 8 July 2002 / Accepted 16 August 2002

\begin{abstract}
The time scale on which chemical peculiarity in upper main sequence stars begins is unknown. Results from the Hipparcos measurements indicate that the classical chemically peculiar $(\mathrm{CP})$ stars occupy the whole area from the zero age main sequence to the terminal age main sequence (Gómez et al. 1998). However, Hubrig et al. (2000) reported that the magnetic fields appear only in stars that have already completed at least approximately $30 \%$ of their main sequence life time. Previous observations in the very young Orion OB1 and Scorpio-Centaurus associations (Joncas \& Borra 1981; Borra et al. 1982) reveal a percentage of chemically peculiar stars that is significantly smaller (less than 5\%) than that of the galactic field (up to $25 \%$ ). This already indicates that the phenomenon needs at least several $10^{6} \mathrm{yr}$ to appear. Since no further observations have been published since then, we have observed the five very young ( $1 \mathrm{Myr} \leq \mathrm{age} \leq 25 \mathrm{Myr}$ ) open clusters Collinder 272, Pismis 20, Lyngå 14, NGC 6396 and NGC 6611. Photometry from 250 CCD frames in the three filter $\Delta a$-system results in very low detection limits ( 0.008 to $0.015 \mathrm{mag}$ ). We find no single photometrically chemically peculiar candidate in approximately 460 observed cluster members in the five program clusters. From the number of observed objects we deduce upper limits between $0.6 \%$ and $2.4 \%$ for the incidence of $\mathrm{CP}$ stars for the four observed aggregates.
\end{abstract}

Key words. stars: chemically peculiar - stars: early-type - techniques: photometric - open clusters and associations: general

\section{Introduction}

For many decades the evolutionary status of chemically peculiar (CP hereafter) stars has been controversial. The results of the Hipparcos mission shed more light on this subject. Gómez et al. (1998) presented the Hertzsprung-Russell-diagram of about $1000 \mathrm{CP}$ stars in the solar neighbourhood using astrometric data from the Hipparcos satellite as well as photometric and radial velocity data. Most $\mathrm{CP}$ stars lie on the main sequence occupying the whole width of it (about 2 mag), just like

Send offprint requests to: E. Paunzen,

e-mail: Ernst.Paunzen@univie.ac.at

* Based on observations obtained at Complejo Astronómico el Leoncito (CASLEO), operated under the agreement between the Consejo Nacional de Investigaciones Científicas y Técnicas de la República Argentina and the National Universities of La Plata, Córdoba y San Juan; ESO-La Silla and UTSO-Las Campanas.

$\star \star$ Member of Carrera del Investigador del Consejo Nacional de Investigaciones Científicas y Técnicas de la República Argentina and Visiting Astronomer at Complejo Astronómico El Leoncito operated under agreement between Consejo Nacional de Investigaciones Cientícas y Técnicas de la República Argentina and the National Universities of La Plata, Córdoba y San Juan. "normal" stars in the same range of spectral types. Their kinematic behaviour is typical of thin disk stars younger than about 1 Gyr.

Hubrig et al. (2000) challenged these results and found that the distribution of the magnetic CP stars of masses below $3 M_{\odot}$ in the Hertzsprung-Russell-diagram differs from that of the "normal" stars in the same temperature range at a high level of significance. Magnetic stars are concentrated toward the center of the main sequence band. In particular, they found that magnetic fields appear only in stars that have already completed at least $30 \%$ of their main sequence life time. No clear picture emerges as to the possible evolution of the magnetic field across the main sequence. Hints of some (loose) relations between magnetic field strength and other stellar parameters are found: stars with shorter periods tend to have stronger fields, as do higher temperature and higher mass stars. A marginal trend of the magnetic flux to be lower in more slowly rotating stars is possible, suggesting a dynamo origin for the magnetic field. No correlation between the rotation period and the fraction of the main sequence life time completed is observed, indicating that the slow rotation in these stars must already have been achieved before they became observably magnetic. 
The first three papers of this series (Bayer et al. 2000; Paunzen \& Maitzen 2001, 2002) deal with 13 intermediate ages and old open clusters. In this paper we present data for five very young open clusters ( $1 \mathrm{Myr} \leq$ age $\leq 25 \mathrm{Myr}$ ) which should help to put tighter constraints on the time scale which the CP phenomenon occurs. Joncas \& Borra (1981) and Borra et al. (1982) presented $\Delta a$-photometry for the Orion OB1 and Scorpio-Centaurus associations. They concluded that the metal enrichment takes place on time scales of a few $10^{7} \mathrm{yr}$ with an incidence of CP stars less than 5\% of all stars in the relevant temperature domain. They only find a small number of $\mathrm{CP}$ stars that exceed a $\Delta a$-value of $0.020 \mathrm{mag}$. From those objects only seven are hotter than A0 (six are assigned as being B9 or B9.5). Since most of the stars at the main sequence for very young open cluster are hotter than $\mathrm{A} 0$, such a low detection percentage already calls for observations of more young and rich aggregates.

\section{Observations, reduction and results}

Observations of the five open clusters were performed with the Bochum $61 \mathrm{~cm}$ (ESO-La Silla), the Helen-SawyerHogg $61 \mathrm{~cm}$ telescope (UTSO-Las Campanas Observatory) and the $2.15 \mathrm{~m}$ telescope at the Complejo Astronómico el Leoncito (CASLEO). The Bochum Telescope was equipped with a nitrogen-cooled Thompson 7882 CCD with $384 \times$ 576 pixels, corresponding to a field-of-view of about $3^{\prime} \times 4^{\prime}$, the Helen-Sawyer-Hogg telescope with a water-cooled PM512 ( $516 \times 516$ pixels) equivalent to $4^{\prime} \times 4^{\prime}$ and the $2.15 \mathrm{~m}$ telescope with a TEK-1024 CCD (using a focal reducer) corresponding to an useful area of $6.7^{\prime}$ on the sky. The observing $\log$ is listed in Table 1. In total, 250 frames for all five clusters in three filters were observed and used for further analysis.

The basic reductions (bias-subtraction, dark-correction, flat-fielding) were carried out within standard IRAF routines. For all frames we have applied a point-spread-function-fitting within the IRAF task DAOPHOT (Stetson 1987). Photometry of each frame was performed separately and the measurements were then averaged and weighted by their individual photometric error.

Since four of our five program clusters (Pismis 20, Lyngå 14, NGC 6396 and NGC 6611) exhibit a significant differential reddening throughout the observed field, we had to correct for it. The normality line (see Bayer et al. 2000 for the definition) is shifted by $E\left(g_{1}-y\right)=0.4 E(B-V)$ to the red and by a small amount $E(a)$ to higher $a$-values (Maitzen 1993)

$a($ corr $)=a($ obs $)-f E(B-V)$.

Assuming a mean $f$-value of 0.05 , we are able to determine an $a$ (corr)-value for each cluster member suffering from differential reddening. As a diagnostic tool, an $a$ (corr) versus $\left(g_{1}-y\right)_{0}$ is used (see Fig. 2). We also show the $V_{0}$ versus $\left(g_{1}-y\right)_{0}$ diagrams for these clusters.

The values of the differential reddening have been taken from the literature (see the corresponding section for references).

The way of calculating the normality line, deriving the errors and calibration of our $y$ measurements, is the same as in
Table 1. Observing log.

\begin{tabular}{lcccccc}
\hline \hline Cluster & Site & Date & $\#_{N}$ & $\#_{g_{1}}$ & $\#_{g_{2}}$ & $\#_{y}$ \\
\hline Cr 272 & CASLEO & 08.2001 & 2 & 11 & 11 & 11 \\
Pismis 20 & CASLEO & 08.2001 & 2 & 30 & 30 & 20 \\
Lyngå 14 & CASLEO & 08.2001 & 2 & 30 & 20 & 20 \\
NGC 6396 & ESO & 04.1995 & 4 & 6 & 7 & 5 \\
& UTSO & 04.1995 & 1 & 2 & 2 & 2 \\
NGC 6611 & ESO & 04.1995 & 4 & 11 & 10 & 11 \\
& UTSO & 04.1995 & 1 & 4 & 3 & 4 \\
\hline
\end{tabular}

previous works (Paunzen \& Maitzen 2001, 2002). We note that the zero points for the measurements taken at CASLEO are different from those of the other two observatories. This is due to different CCD gain and bias levels.

Table 2 lists our observed open clusters and their characteristics from the literature (as listed in the corresponding sections). Furthermore, the number of observed stars, the number of individual frames (Table 1), the regression coefficients for all transformations and normality lines as well as the $3 \sigma$ detection limits are given.

The finding charts of our open clusters are shown in Fig. 1. The size of the symbols (by area) is inversely proportional to the apparent visual magnitudes of the objects in the sense that larger symbols denote brighter objects.

The complete Tables 4 to 8 with all data for the individual cluster stars are available in electronic form at the CDS via anonymous ftp to cdsarc.u-strasbg.fr (130.79.125.5) or via http://cdsweb.u-strasbg.fr/cgi-bin/qcat?J/A+A/395/823 or upon request from the first author. These tables include the cross identification of objects from the literature, the observed $\left(g_{1}-y\right)$ and $a$ values with their corresponding errors, $V$ magnitudes, the $(B-V)$ values from the literature, $\Delta a$-values derived from the normality lines of $\left(g_{1}-y\right),(B-V)$, respectively, as well as the reddening values $E(B-V)$ for the clusters Pismis 20, Lyngå 14, NGC 6396 and NGC 6611.

The diagnostic diagrams for all five open clusters are shown in Fig. 2. Furthermore, the normality lines and the confidence intervals corresponding to $99.9 \%$ are plotted.

\subsection{Collinder 272}

Two papers were devoted so far to this open cluster: Fenkart et al. (1977) presented $R G U$ measurements and Vázquez et al. (1997) list results from an extensive CCD UBVRI photometric survey. Both sources agree very well on the age of Collinder 272 (13 Myr) but disagree concerning distance. Vázquez et al. (1997) give a distance of $2300 \pm 200$ pc using $R=3.1$ or 3200 pc for $R=3.4$, respectively. Fenkart et al. (1977) give a distance of $2900 \mathrm{pc}$. For our purpose, we have taken a mean value of $2600 \mathrm{pc}$.

Our observations are centered on the northern area of this cluster ( $Y \geq 900$ in Fig. 2 of Vázquez et al. 1997). Although the $\Delta a$-detection limit is very low $(0.010 \mathrm{mag})$, we find no peculiar objects (Fig. 2). Because of the very young age of Collinder 272, almost all stars in the relevant temperature 


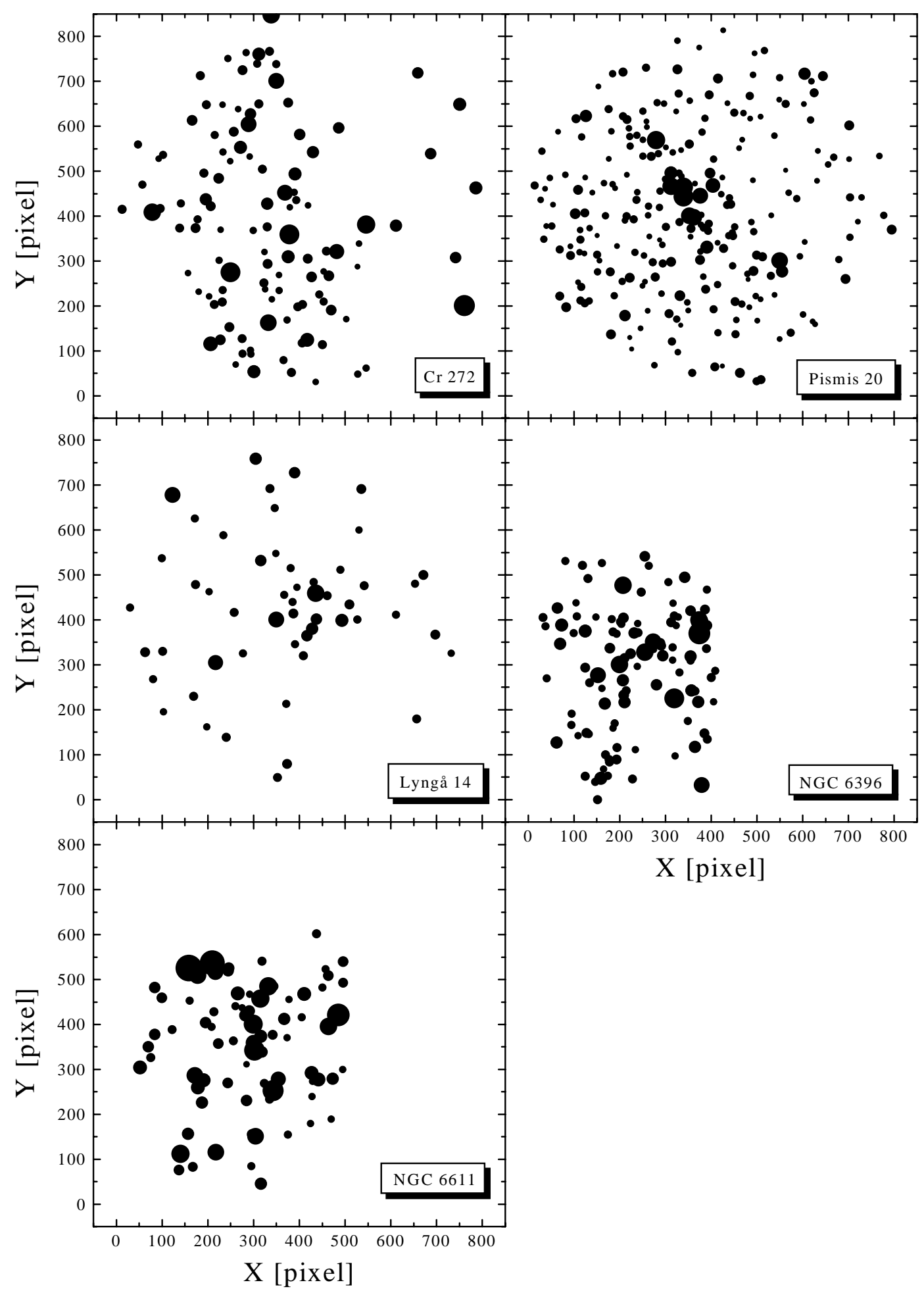

Fig. 1. Finding charts for the program clusters. North is to the right and west is upwards; 1 pixel $=0.5^{\prime \prime}$. The sizes (by area) of the open circles are inversely proportional to the $V$-magnitudes taken from Tables 4 to 8 larger open circles denote brighter objects.

domain for the $\mathrm{CP}$ phenomenon have to be still at their pre-main sequence evolution.

\subsection{Pismis 20}

This open cluster (age $\approx 5 \mathrm{Myr}$ ) includes one hypergiant (HD 134959, BIa-0) and Wolf-Rayet star (WR 67), respectively. The differential reddening is rather strong throughout the cluster area $(1.0<E(B-V)<1.5 \mathrm{mag}$; Turner 1996; Sagar et al. 2001) which is due the large distance (3500 pc; Vázquez et al. 1995; Turner 1996; Sagar et al. 2001) and its low galactic latitude $\left(-1^{\circ}\right)$. We have used the reddening maps of Turner (1996) and Sagar et al. (2001) to construct our diagnostic diagrams (Fig. 2). 

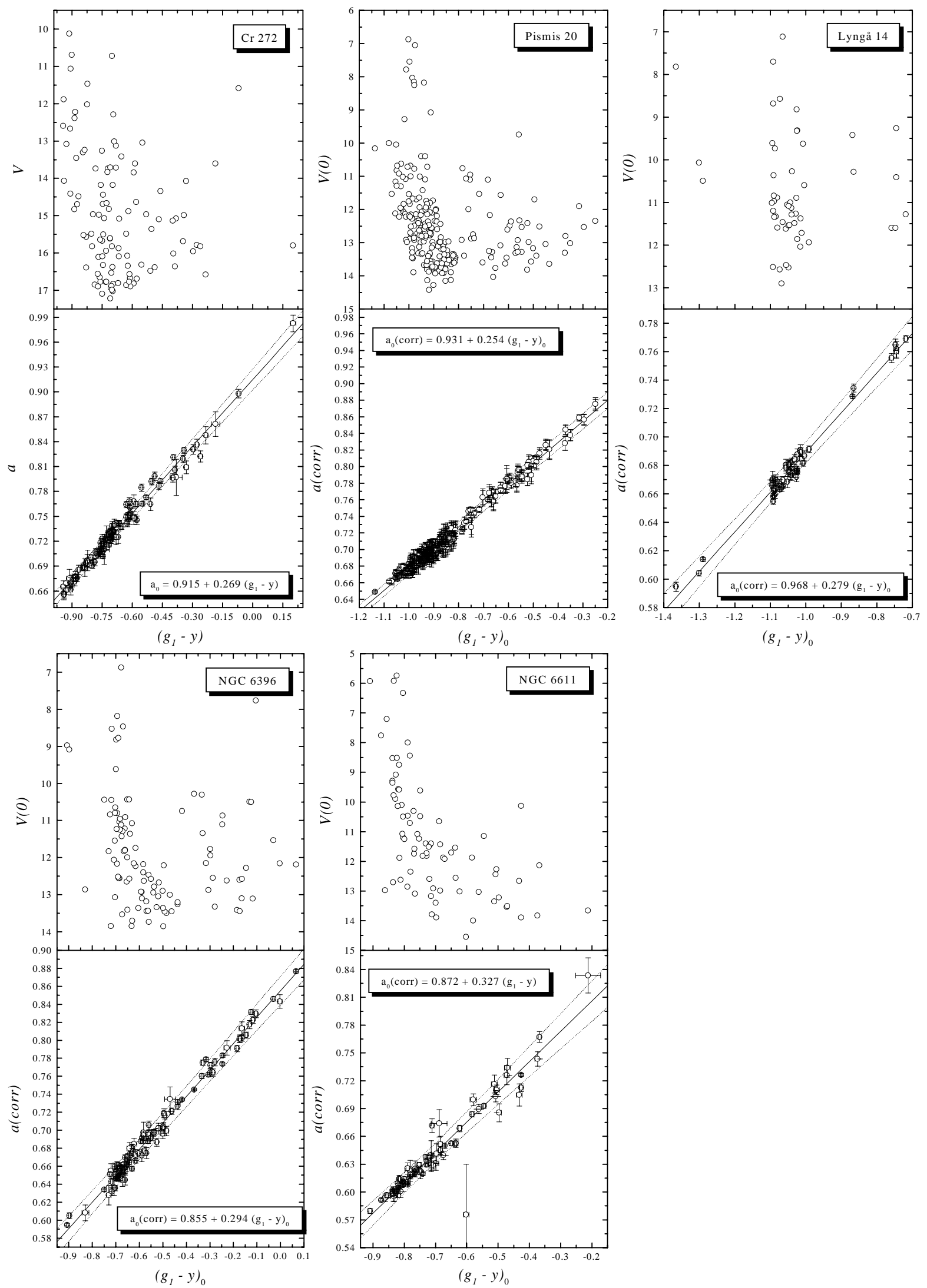

Fig. 2. Observed diagrams for our program clusters. The solid line is the normality line whereas the dotted lines are the confidence intervals corresponding to $99.9 \%$. The error bars for each individual object are the mean errors. The measurement errors of $V$ are much smaller than the symbols and have been omitted. 
Table 2. Summary of results. The errors in the final digits of the corresponding quantity are given in parenthesis.

\begin{tabular}{|c|c|c|c|c|c|}
\hline Name & $\begin{array}{c}\text { Cr } 272 \\
\text { C1327-610 }\end{array}$ & $\begin{array}{c}\text { Pismis } 20 \\
\text { C1511-588 }\end{array}$ & $\begin{array}{c}\text { Lyngå } 14 \\
\text { C1651-452 }\end{array}$ & $\begin{array}{l}\text { NGC 6396 } \\
\text { C1734-349 }\end{array}$ & $\begin{array}{l}\text { NGC 6611 } \\
\text { C1816-138 }\end{array}$ \\
\hline$l / b$ & $308 /+1$ & $321 /-1$ & $341 /-1$ & $354 /-2$ & $17 /+1$ \\
\hline$E(B-V)$ & 0.45 & 1.24 (var) & 1.48 (var) & 0.96 (var) & 0.86 (var) \\
\hline$d[\mathrm{pc}]$ & 2600 & 3500 & 2300 & 1300 & 2100 \\
\hline $\log t$ & 7.11 & 6.70 & 6.00 & 7.40 & 6.48 \\
\hline Tr-type & III $2 \mathrm{~m}$ & I $3 p$ & II $1 \mathrm{p}$ & II $3 \mathrm{~m}$ & II $3 \mathrm{~m}$ \\
\hline$n(\mathrm{obj})$ & 111 & 238 & 53 & 105 & 79 \\
\hline$V=a+b \cdot(y)$ & $-4.97(1) 0.998(1)$ & $-4.84(9) 0.998(4)$ & $-4.97(35) 1.000(19)$ & $-6.75(62) 0.930(30)$ & $-8.00(12) 1.000(3)$ \\
\hline$a_{0}=a+b \cdot\left(g_{1}-y\right)$ & $0.915(3) 0.269(4)$ & & & & \\
\hline$a_{0}=a+b \cdot\left(g_{1}-y\right)_{0}$ & & $0.931(2) 0.254(2)$ & $0.968(6) 0.279(6)$ & $0.855(2) 0.294(4)$ & $0.872(7) 0.327(10)$ \\
\hline$a_{0}=a+b \cdot(B-V)$ & $0.643(6) 0.098(5)$ & & & & \\
\hline$a_{0}=a+b \cdot(B-V)_{0}$ & & $0.707(1) 0.102(2)$ & & & $0.634(3) 0.115(7)$ \\
\hline $3 \sigma[\mathrm{mag}]$ & 0.010 & 0.008 & 0.010 & 0.014 & 0.015 \\
\hline$n$ (frames) & 33 & 80 & 70 & 24 & 43 \\
\hline
\end{tabular}

The following objects have inconsistent $(B-V)$ values in Vázquez et al. (1995) and Sagar et al. (2001): WEBDA-number 1058, 1086, 1088, 1089, 1092, 1094, 1099, 1108, 1129, 1138 and 1159 . We are not able to verify the error source since no other independent measurements for these objects exist.

A total of 80 frames give a detection limit of 0.008 mag for Pismis 20. The main sequence is rather broad due to the fact that most members are just arriving at the zero age main sequence. There are also many cooler objects which are all probably non-members. No single candidate CP star was detected from 238 objects measured, the magnitude range for members is almost eight magnitudes.

\subsection{Lyngå 14}

The earliest members of this open cluster have a spectral type of O9 (Moffat \& Vogt 1975) which suggests an age of about 1 Myr. The only published photometry is by Moffat \& Vogt (1975). Orsatti et al. (1998) presented polarimetry for eleven stars in the cluster area. The distance of Lyngå 14 from the Sun is about $2300 \mathrm{pc}$ with a mean reddening of $E(B-V)=1.48 \mathrm{mag}$. The photometric measurements were dereddened using the values given in Orsatti et al. (1998). The main sequence is well defined according to Fig. 2, containing about 40 objects with spectral types (derived from the photometry) earlier than B9. However, the true membership of the bona-fide cluster stars has to be proven by further photometric measurements in the Johnson $U B V$ and Strömgren uvby systems. We find no CP candidates within this cluster above the given detection limit (0.010 mag).

\subsection{NGC 6396}

The only published data for this cluster are listed in Moffat \& Vogt (1975). They presented Johnson $U B V$ photometry together with estimates of the differential reddening. According to them, NGC 6396 has a distance of 1300 pc and an earliest
Table 3. $\Delta a$-values for known Be stars of NGC 6611. The numbers are according to Walker (1961), the spectral types were taken from Walker (1961) and Hillenbrand et al. (1993).

\begin{tabular}{cccl}
\hline \hline No. & $\Delta a\left(g_{1}-y\right)_{0}$ & $\Delta a(B-V)_{0}$ & Spec. \\
\hline 210 & +0.000 & -0.002 & B3 Ve \\
221 & +0.003 & +0.003 & B8 Ve \\
243 & -0.012 & +0.009 & B8 Ve \\
323 & +0.003 & +0.002 & B5 Ve \\
\hline
\end{tabular}

spectral type of B3 which corresponds to a turn-off color of $(B-V)_{\mathrm{t}}=-0.20 \mathrm{mag}$ (age about $25 \mathrm{Myr}$ ). The main sequence comprises about 80 stars and is clearly separated from backas well as foreground objects. Again, no CP candidates were detected.

\subsection{NGC 6611}

This open cluster has been extensively studied in the past. Belikov et al. (1999) summarize the results of the past 30 years and served us as database. NGC 6611 is known as part of a star-forming region near the external border of the SagittariusCarina spiral arm at a distance of $2100 \mathrm{pc}$ and an age of about 3 Myr. It is probably associated with the bright emission nebula M 16 (the Eagle Nebula) and suffers from strong differential reddening. We have observed 79 stars photometrically in order to find new CP candidates. Already Walker (1961) had found that the main sequence extends from O5 to B5 with cooler pre-main sequence objects still above it. We find two objects (No. 198 and 229 in the numbering system of Walker 1961) that show a peculiar $\Delta a$. The values are $\Delta a\left(g_{1}-y\right)_{0}=+0.027$ and +0.034 mag as well as $\Delta a(B-V)_{0}=+0.061$ and 0.016 mag for No. 198 and 229, respectively. According to Belikov et al. (1999) No. 198 is certainly a non-member whereas No. 229 has a membership probability of only $30 \%$. The Johnson $U B V$ colors for No. 229 are also very unusual: $(B-V)_{0}=+0.204 \mathrm{mag}$, 
Table 4. The upper limits for the incidence of CP stars in our program clusters. The number of observed members has been deduced from Fig. 2; the incidence was derived assuming that one (undetected) $\mathrm{CP}$ exists for the observed number of members.

\begin{tabular}{cccc}
\hline \hline Cluster & upper limit & No. (members) & Incidence \\
\hline Cr 272 & 0.010 & 95 & $<1.1 \%$ \\
Pismis 20 & 0.008 & 180 & $<0.6 \%$ \\
Lyngå 14 & 0.010 & 40 & $<2.4 \%$ \\
NGC 6396 & 0.014 & 80 & $<1.3 \%$ \\
NGC 6611 & 0.015 & 65 & $<1.6 \%$ \\
\hline
\end{tabular}

$(U-B)_{0}=-0.552 \mathrm{mag}$ and $M_{\mathrm{V}}=+2.15 \mathrm{mag}$. We therefore believe that this object is an undetected spectroscopic binary system. One star (No. 36 according to our numbering system) has an extreme negative $\Delta a$-value of $-0.099 \mathrm{mag}$ but since the measurement error is $0.054 \mathrm{mag}$, it is not significant. There are also four known Be stars measured within our photometric system. Pavlovski \& Maitzen (1989) have shown that only very peculiar Be stars during their shell phase (e.g. Pleione) mimick a classical CP star with a significant positive $\Delta a$-value. Otherwise, Be stars tend to show only small negative deviations from the normality line. In Table 3 we present our results for four Be stars in NGC 6611. Our measurements are well in line with the findings of Pavlovski \& Maitzen (1989) which were derived for galactic field stars only.

\section{The incidence of very young CP stars}

From our observations we are able to give an upper limit of CP stars in the relevant young open clusters. Table 4 lists the upper limits for the incidence of CP stars in the five program clusters. The number of observed members has been deduced from Fig. 2 and the incidence was derived assuming that one (undetected) CP exists for the observed number of members. It is impossible to deduce the true membership for all observed cluster stars. When possible, we have checked the literature for membership probabilities and further two color diagrams. However, from a comparison with the published literature we conclude that the derived percentages are representative at $\pm 1 \%$. We find upper limits between $0.6 \%$ and $2.4 \%$ for a sample of 460 stars observed. Joncas \& Borra (1981) and Borra et al. (1982) concluded from $\Delta a$-photometry for the Orion OB1 and Scorpio-Centaurus associations that the incidence of CP stars is less than 5\% for these associations. Notice that the overall percentage of classical CP stars peaks at a spectral type of A0 with a maximum of $25 \%$ for galactic field stars.

\section{Conclusions}

We find no photometrically $\mathrm{CP}$ star in the very young (1 Myr $\leq$ age $\leq 25 \mathrm{Myr}$ ) open clusters Collinder 272, Pismis 20, Lyngå 14, NGC 6396 and NGC 6611. This result is based on photometry from $250 \mathrm{CCD}$ frames in the three filter $\Delta a$ system. The very low detection limits $(0.008$ to $0.015 \mathrm{mag})$ together with the high number of observed stars (approximately 460 cluster members in all five program clusters) makes this null result even more significant. From the number of observed objects we deduce upper limits between $0.6 \%$ and $2.4 \%$ for the incidence of CP stars. This is in line with previous observations for the Orion OB1 and Scorpio-Centaurus associations (upper limits of about 5\%). This favours the theory that the CP phenomenon needs at least several Myr to start being effective.

Acknowledgements. The authors acknowledge use of the CCD and data acquisition system supported under U.S. National Science Foundation grant AST-90-15827 to R.M. Rich. This work benefitted from the financial contributions of the City of Vienna (Hochschuljubiläumsstiftung project: Wiener Zweikanalphotometer). EP acknowledges partly support by the Fonds zur Förderung der wissenschaftlichen Forschung, project P14984. Use was made of the SIMBAD database, operated at CDS, Strasbourg, France and the WEBDA database, operated at the Institute of Astronomy of the University of Lausanne.

\section{References}

Bayer, C., Maitzen, H. M., Paunzen, E., Rode-Paunzen, M., \& Sperl, M. 2000, A\&AS, 147, 99 (Paper I)

Belikov, A. N., Kharchenko, N. V., Piskunov, A. E., \& Schilbach, E. 1999, A\&AS, 134, 525

Fenkart, R. P., Binggeli, D., Good, D., et al. 1977, A\&AS, 30, 307

Gómez, A. E., Luri, X, Grenier, S., et al. 1998, A\&A, 336, 953

Hillenbrand, L. A., Massey, P., Strom, S. E., \& Merrill, K. M. 1993, AJ, 106, 1906

Hubrig, S., North, P., \& Mathys, G. 2000, ApJ, 539, 352

Maitzen, H. M. 1993, A\&AS, 199, 1

Moffat, A. F. J., \& Vogt, N. 1975, A\&AS, 20, 155

Orsatti, A. M., Vega, E., \& Marraco, H. G. 1998, AJ, 116, 266

Paunzen, E., \& Maitzen, H. M. 2001, A\&A, 373, 153 (Paper II)

Paunzen, E., \& Maitzen, H. M. 2002, A\&A, 385, 867 (Paper III)

Pavlovski, K., \& Maitzen, H. M. 1989, A\&AS, 77, 351

Sagar, R., Munari, U., \& de Boer, K. S. 2001, MNRAS, 327, 23

Stetson, P. B. 1987, PASP, 99, 191

Turner, D. G. 1996, AJ, 111, 828

Vázquez, R. A., Will, J.-M., Prado, P., \& Feinstein, A. 1995, A\&AS, 111,85

Vázquez, R. A., Baume, G., Feinstein, A., \& Prado, P. 1997, A\&AS, 124, 13

Walker, M. F. 1961, ApJ, 133, 438 